\title{
Latest CT technologies in lung cancer screening: protocols and radiation dose reduction
}

\author{
Marleen Vonder ${ }^{1}$, Monique D. Dorrius ${ }^{1,2}$, Rozemarijn Vliegenthart ${ }^{2}$ \\ ${ }^{1}$ Department of Epidemiology, University of Groningen, University Medical Center Groningen, Groningen, The Netherlands; ${ }^{2}$ Department of \\ Radiology, University of Groningen, University Medical Center Groningen, Groningen, The Netherlands \\ Contributions: (I) Conception and design: All authors; (II) Administrative support: None; (III) Provision of study materials or patients: None; (IV) \\ Collection and assembly of data: None; (V) Data analysis and interpretation: None; (VI) Manuscript writing: All authors; (VII) Final approval of \\ manuscript: All authors. \\ Correspondence to: Prof. Dr. Rozemarijn Vliegenthart, MD, PhD. Department of Radiology, EB44, University Medical Center Groningen, Hanzeplein \\ 1, 9713 GZ Groningen, The Netherlands. Email: r.vliegenthart@umcg.nl.
}

\begin{abstract}
The aim of this review is to provide clinicians and technicians with an overview of the development of CT protocols in lung cancer screening. CT protocols have evolved from pre-fixed settings in early lung cancer screening studies starting in 2004 towards automatic optimized settings in current international guidelines. The acquisition protocols of large lung cancer screening studies and guidelines are summarized. Radiation dose may vary considerably between CT protocols, but has reduced gradually over the years. Ultra-low dose acquisition can be achieved by applying latest dose reduction techniques. The use of low tube current or tin-filter in combination with iterative reconstruction allow to reduce the radiation dose to a submilliSievert level. However, one should be cautious in reducing the radiation dose to ultra-low dose settings since performed studies lacked generalizability. Continuous efforts are made by international radiology organizations to streamline the CT data acquisition and image quality assurance and to keep track of new developments in CT lung cancer screening. Examples like computer-aided diagnosis and radiomic feature extraction are discussed and current limitations are outlined. Deep learning-based solutions in postprocessing of CT images are provided. Finally, future perspectives and recommendations are provided for lung cancer screening CT protocols.
\end{abstract}

Keywords: Lung cancer; computed tomography; screening; radiation dose reduction

Submitted Jul 03, 2020. Accepted for publication Oct 22, 2020.

doi: $10.21037 /$ tlcr-20-808

View this article at: http://dx.doi.org/10.21037/tlcr-20-808

\section{Introduction}

A growing number of countries is implementing lung cancer screening programs or are at the forefront of starting with research implementation studies (1-7). The National Lung Screening trial (NLST, USA) and the NederlandsLeuvens Longkanker Screenings Onderzoek (NELSON) trial have shown that lung cancer mortality can be reduced with at least $20 \%$ with lung cancer CT screening in high risk individuals meeting eligibility criteria $(8,9)$. Current lung cancer screening protocols recommend repeated screening at either annual or biannual frequency for eligible individuals $(10,11)$. On an individual level this may result in over 25 scans over a lifetime with in practice widely varying cumulative radiation dose $(12,13)$. Consequently, the estimates for induced cancer risk by repeated screening for an entire population vary as well depending on the used CT protocol, CT generation and expertise of institutions to optimize CT protocols (12-15). The aim of this review is to provide clinicians with an overview of the development of the CT protocol for lung cancer screening, the current protocol recommendations and radiation dose reduction possibilities in lung cancer CT screening. 


\section{CT protocol settings of large lung cancer screening studies}

A variety of CT systems and low dose CT acquisition protocols have been used in large lung cancer screening studies, see Table 1 . The majority of studies were initiated before 2010, and therefore, the protocol settings (radiation dose and image quality) dates back to systems and standards of ten until twenty years ago. Lung cancer CT screening studies before 2004 used single slice detector systems or the first multi(slice)-detector systems that could acquire 4 slices per rotation, and used collimation of $2.5-5.0 \mathrm{~mm}$ (16-18). From the start, a spiral scan mode was used to cover the entire chest from lung apices to lung base within one end-inspiratory breath hold. Starting from studies initiated in 2004, 16-slice multi-detector CT systems were mainly used in lung cancer screening from then on. This allowed acquiring 16 slices with collimation of $0.75 \mathrm{~mm}$ resulting in thinner axial slices $(19,23,24)$. From 2007 onwards, number of slices in MDCT increased to 128 with collimation as low as $0.625 \mathrm{~mm}$, and scan times ranging from 5 to 10 seconds $(26,27)$.

The tube voltage used in the major screening studies varied between 80 and $140 \mathrm{kVp}$. In general, either one fixed tube voltage and/or tube current value was applied on each CT system for every participant or pre-defined values were applied based on a participant's body weight. For instance, a low tube voltage of $80 \mathrm{kVp}$ was used in slim or small participants (up to $50 \mathrm{~kg}$ ), moderate tube voltage of $120 \mathrm{kVp}$ in medium size participants (between $50-80 \mathrm{~kg}$ ) and a high tube voltage of $140 \mathrm{kVp}$ in large or tall participants (over $80 \mathrm{~kg}$ ). Similarly, the tube current was mainly fixed at a low current of maximum $40 \mathrm{~mA}$ to achieve a low dose chest CT, or the tube current was adjusted in small, medium and large participants to reach predefined total radiation dose as estimated by the volume CT dose index $\left(\mathrm{CTDI}_{\mathrm{vol}}\right)$ in mGy (23). As far as results on total mean radiation dose have been published, overall the radiation dose of the low-dose chest protocols varied between 0.8 to $1.5 \mathrm{mSv}$ for medium size participants in the trials initiated between 2001 and $2011(17,20,21,25,26)$. To put into context, the radiation dose of conventional high resolution CT of the chest used for clinically indicated scans, could be as high as $5.8 \mathrm{mSv}$ at that time (28).

The specific reconstruction values of the various study protocols are scarcely published. Information on used fieldof-view, reconstruction algorithm or window settings are not published. In general, filtered back projection (FBP) was used to reconstruct the images at a thin slice thickness and a small increment. This resulted in overlapping slice reconstructions to increase the image quality without the need of increasing radiation dose, to acquire thin slices for the detection of small lung nodules. In the process of reconstructing the image with FBP, a convolution filter or 'kernel' is used to enhance or optimize the reconstructed image for specific anatomical areas. In the lung cancer screening studies, a 'soft' kernel and 'hard' or 'sharp' kernel was applied. See Figure 1 for example of soft versus sharp kernel CT image. Soft kernels are optimized for visualizing soft tissues, appear as more smooth images with less noise, and are used to detect nodules and evaluate nodule morphology. Sharper kernels are suitable for visualizing high differences in density (lung-air, bonesoft tissue) structures, appear as sharp images with higher spatial resolution at the expense of higher noise, and can be used for nodule size measurements although segmentation problems may occur. A soft kernel tends to show higher reproducibility for nodule volume measurements but may underestimate nodule volume. There is no consensus yet for the use of soft or sharp kernel $(22,29)$, in general, a medium-smooth to medium-sharp kernel is advised (30). It is of particular importance to consistently use the same reconstruction kernel in case of nodule measurements on subsequent CT scans.

\section{International guidelines and recommendations}

Several international radiology societies have published guidelines on the CT requirements for lung cancer screening, see Table 2, whereas the guidelines of thoracic societies mainly focus on the analysis of the CT images and lung nodule management.

The American College of Radiology (ACR), Society of Thoracic Radiology (STR) and the European Society of Thoracic Imaging (ESTI) have published practice guidelines and technical standards to assist radiologists and medical physicists in developing local CT lung cancer screening protocols (31-33). The ACR recommends using at least 16-slice MDCT, whereas the ESTI sets the lower limit at 32-slice and recommends 64-slice MDCT. The total scan time is recommended to be below 10 seconds to cover the total chest within a single breathhold. A tube voltage of $100-120 \mathrm{kVp}$ is acceptable for standard sized patients, whereas a tube voltage of $140 \mathrm{kVp}$ may be used in obese patients. The tube current should be set in conjunction with the tube voltage and pitch to meet certain $\mathrm{CTDI}_{\mathrm{vol}}$. 


\begin{tabular}{|c|c|c|c|c|c|c|c|c|}
\hline 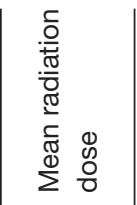 & 1 & 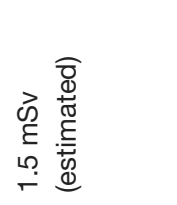 & 1 & 1 & 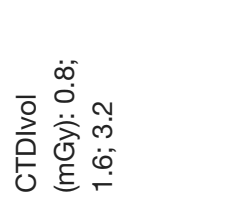 & 1 & $\begin{array}{l}\text { के } \\
\text { है } \\
0 \\
\text { जे } \\
\stackrel{0}{+}\end{array}$ & 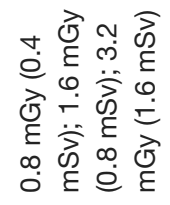 \\
\hline 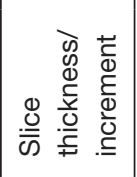 & $\begin{array}{l}\xi \\
\xi \\
\stackrel{\xi}{\rho}\end{array}$ & 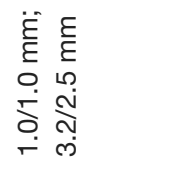 & 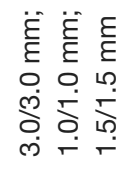 & 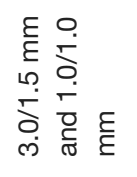 & 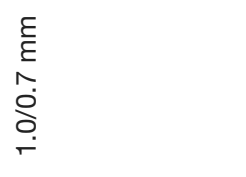 & 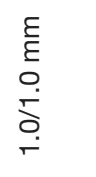 & 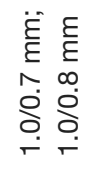 & $\begin{array}{l}\varepsilon \\
\varepsilon \\
\hat{\rho} \\
\stackrel{0}{0} \\
\stackrel{r}{-}\end{array}$ \\
\hline $\begin{array}{l}\bar{\Phi} \\
\stackrel{\bar{D}}{\Phi} \\
\stackrel{\Sigma}{\underline{T}}\end{array}$ & 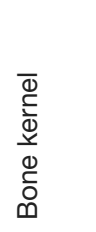 & 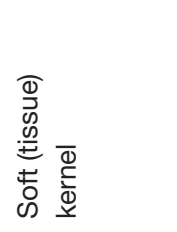 & 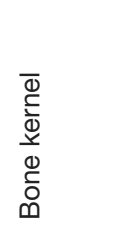 & 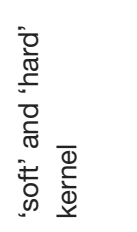 & 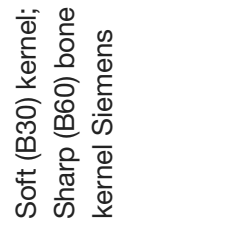 & 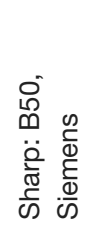 & & 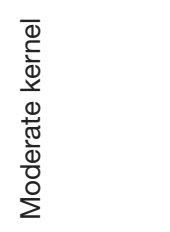 \\
\hline 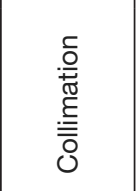 & $\begin{array}{l}\varepsilon \\
\varepsilon \\
\underline{E} \\
x \\
- \\
-\end{array}$ & $\begin{array}{l}\varepsilon \\
\xi \\
\llcorner \\
\tilde{v}\end{array}$ & 1 & $\begin{array}{l}\varepsilon \\
\varepsilon \\
\underline{L} \\
\stackrel{1}{0} \\
\hat{0} \\
\dot{x} \\
\underline{0}\end{array}$ & 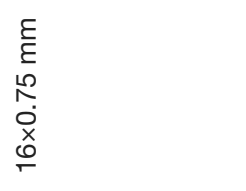 & 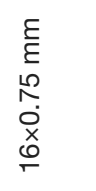 & 1 & 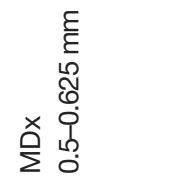 \\
\hline 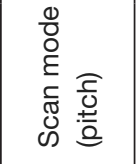 & $\begin{array}{l}\overline{\overline{0}} \\
\overline{\overline{\overline{0}}}\end{array}$ & $\begin{array}{l}\overline{\overline{0}} \\
\overline{\overline{\overline{0}}} \\
\dot{\omega}\end{array}$ & $\begin{array}{l}\overline{\overline{0}} \\
\overline{\overline{\overline{0}}}\end{array}$ & 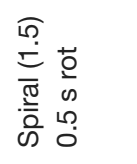 & 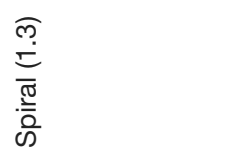 & 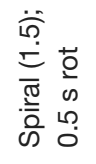 & 1 & 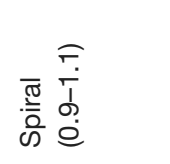 \\
\hline 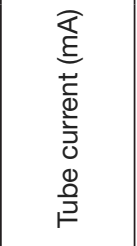 & $\begin{array}{l}\varangle \\
\xi \\
o\end{array}$ & 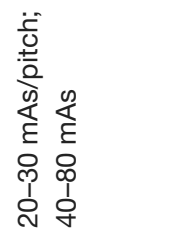 & 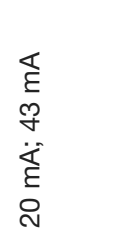 & 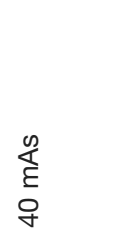 & 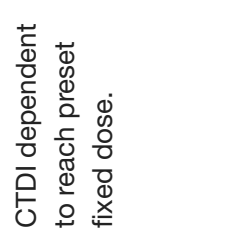 & 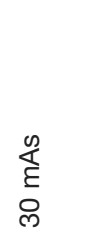 & 1 & 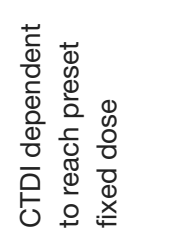 \\
\hline 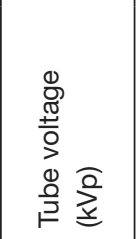 & $\begin{array}{l}\stackrel{0}{2} \\
\text { o } \\
\frac{9}{1}\end{array}$ & 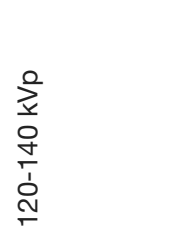 & 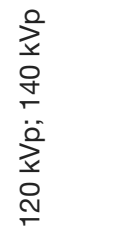 & 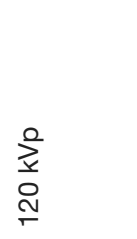 & 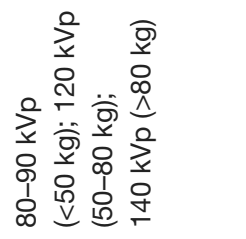 & $\begin{array}{l}\stackrel{0}{2} \\
\stackrel{\text { N}}{N}\end{array}$ & । & 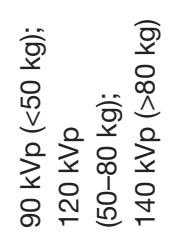 \\
\hline 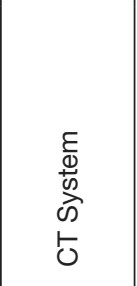 & $\begin{array}{l}5 \\
\text { क }\end{array}$ & 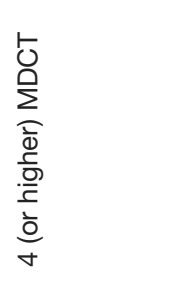 & 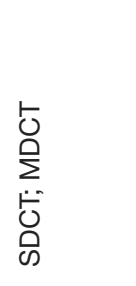 & 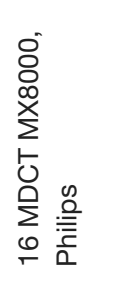 & 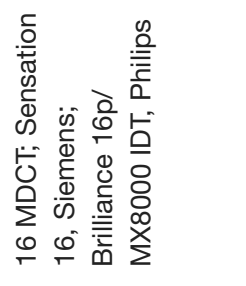 & 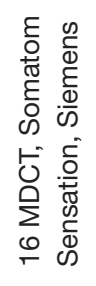 & 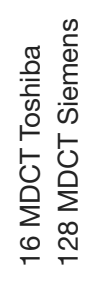 & 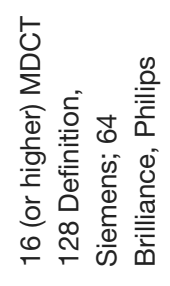 \\
\hline 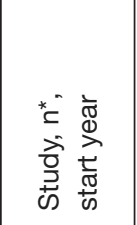 & 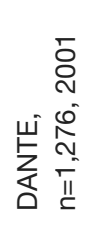 & 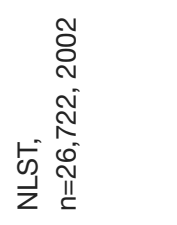 & 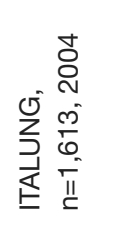 & 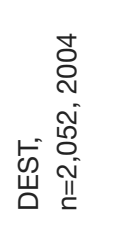 & 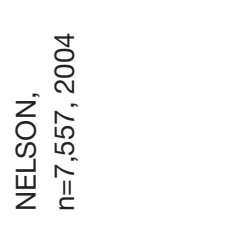 & 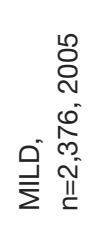 & 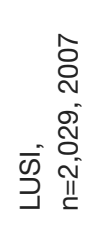 & 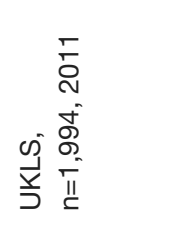 \\
\hline 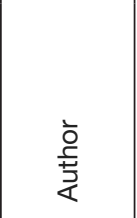 & 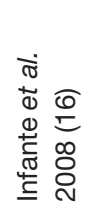 & 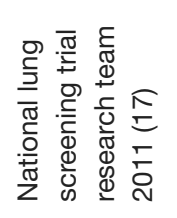 & 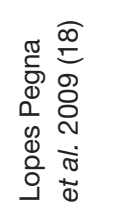 & 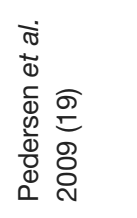 & 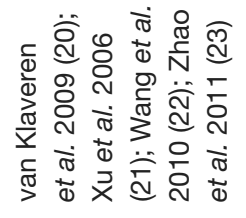 & 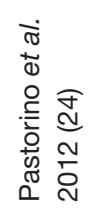 & 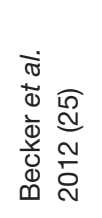 & 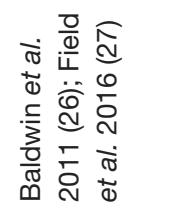 \\
\hline
\end{tabular}




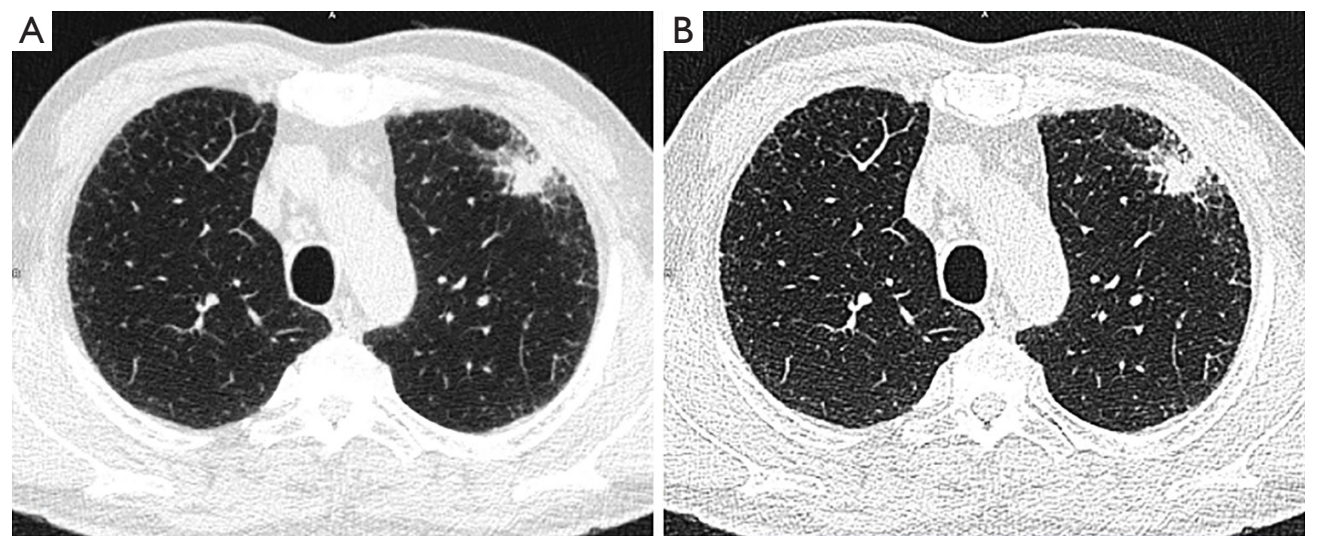

Figure 1 Example of low dose CT protocol with $120 \mathrm{kVp}$ and FBP reconstructed with (A) soft and (B) hard kernel in a patient presenting with an irregular solid nodule of $3,973 \mathrm{~mm}^{3}$ (maximum diameter $26.7 \mathrm{~mm}$ ) in the left upper lobe.

Beam-hardening pre-filters like tin-filter $(\mathrm{Sn})$ may be applied if available on the CT system (33). The guidelines discourage the use of fixed tube currents, but highly recommend the use of automated tube current modulation based on a patient's habitus. Moreover, the use of automated tube voltage selection and organ dose modulation is advised. The ACR recommends a pitch between 0.7-1.5, but states that this parameter should be set with the other acquisition parameters mentioned before and the CTDI $\mathrm{vol}_{\mathrm{vo}}$. This pitch factor is inherent to the system settings since it is based on the rotation time, table feed and output (beam width) of a CT system. The CTDI ${ }_{\text {vol }}$ to be met decreased over the years, the ACR in 2014 reports a level of $<3$ mGy for standard patient size, while the ESTI 2019 reports levels of $<1.6 \mathrm{mGy}$ for patient size $>80 \mathrm{~kg}$, resulting in an effective radiation dose of approximately $0.7 \mathrm{mSv}$.

In contrast to the former studies, the current guidelines report in detail on the reconstruction parameters to be used. Reconstruction of the CT images is preferred at slice thickness $\leq 1.0 \mathrm{~mm}$ and a slice increment smaller than the slice thickness $(\leq 0.7 \mathrm{~mm})$, but overlapping reconstructions are not mandatory. The field-of-view may be optimized for every patient to include the entire lungs up to $1 \mathrm{~cm}$ beyond the rib cage. Standard body or mediastinum/soft tissue and lung kernels should be used and additionally a medium-sharp (lung) kernel without edge enhancement may be used. Moreover, during analysis it is advised to use maximum intensity projections (MIP) and multiplanar reconstruction (MPR) for lung nodule detection and nodule characterization respectively, see Figure 2. Both guidelines strongly recommend the use of iterative reconstruction or deep learning reconstruction to allow further dose reduction while maintaining image quality. The ESTI guideline discourages the use of FBP (33).

In addition to the radiological societies, a working group of the American Association of Physicists in Medicine (AAPM) developed a set of detailed acquisition protocols of over $30 \mathrm{CT}$ systems of six major vendors for lung cancer screening purposes (34). These protocols are based on the experience gained with the NLST study and other screening studies by the working group. Similar to the ACR guideline, these protocols should result in radiation dose (CTDIvol) $\leq 3 \mathrm{mGy}(\leq 1.0 \mathrm{mSv})$ for a standardized patient of $70 \mathrm{~kg}$. However, radiation dose may vary from 0.25 to $5.6 \mathrm{mGy}$ for patient of 50 to $120 \mathrm{~kg}$.

Other societies provide limited information on CT protocol requirements, but they highlight the importance of using thin slices $(<1.25 \mathrm{~mm})$ and MPR, or MIP for the detection and quantification and characterization of lung nodules and using a low dose technique (11,35-37). Specifically, the Fleischner Society addresses that small nodules $(<10 \mathrm{~mm}$ in diameter) should be quantified on sharp (high spatial resolution) kernel while in larger nodules $(>10 \mathrm{~mm})$ the reconstruction kernel has less impact on measurement accuracy (38).

\section{Radiation dose reduction}

In the past 5 years, multiple studies have evaluated the impact of lowering the radiation further from LDCT to ultra-low dose CT (ULDCT) with submilliSievert dose level. Common applied dose reduction techniques are reducing the tube output by decreasing the tube current and/or tube voltage or by using beam filtration with a tin- 
Table 2 International radiological society's CT protocol guidelines

\begin{tabular}{|c|c|c|}
\hline & ACR - STR [Kazerooni et al. 2019 (31); ACR 2014 (32)] & ESTI [Revel et al. ESTI 2019 (33)] \\
\hline Rotation time & $\leq 75 \mathrm{~ms}$ & $\leq 50 \mathrm{~ms}$ \\
\hline Pitch & $0.7-1.5^{\star}$ & As suggested by vendors* \\
\hline Scan duration & Scan time $<15 \mathrm{~s}$ (single breathhold) & $\leq 10 \mathrm{~s}$ (shorter prefered, single breath hold) \\
\hline \multirow[t]{2}{*}{ Tube voltage } & $100-140 \mathrm{kVp}{ }^{*}$ for standard sized patient & $\begin{array}{l}100-120 \mathrm{kVp} \text { for standard sized patient; } 140 \mathrm{kVp} \text { for } \\
\text { obese participant }\end{array}$ \\
\hline & $\begin{array}{l}\text { kVP should be set in combination with mAs to meet } \\
\text { CTDI }_{\text {vol }} \text { specifications }\end{array}$ & $\begin{array}{l}\text { Preferably reduce } \mathrm{mAs} \text { first and then } \mathrm{kVp} \\
\text { If available: beam-hardening pre-filtering with } \mathrm{Sn} \text { filter is } \\
\text { strongly advised }\end{array}$ \\
\hline Radiation dose $\left(C T D I_{\text {vol }}\right)$ & $\leq 3 \mathrm{mGy}$ for standard patient & $\begin{array}{l}\text { Depending on patient weight: }<50 \text { kg, } 0.4 \text { mGy; } \\
50-80 \mathrm{~kg}, 0.8 \mathrm{mGy} ;>80 \mathrm{~kg}, 1.6 \mathrm{mGy}\end{array}$ \\
\hline FOV & $\begin{array}{l}\text { Optimized for each patient: } 1-\mathrm{cm} \text { beyond rib cage; } \\
\text { does not need to include entire chest wall thickness }\end{array}$ & Does not need to include entire chest wall thickness \\
\hline Slice thickness & $\leq 2.5 \mathrm{~mm}$ slice thickness, $\leq 1.0 \mathrm{~mm}$ preferred & $\begin{array}{l}\leq 1.0 \mathrm{~mm}, \leq 0.75 \mathrm{~mm} \text { preferred, } 1.25 \mathrm{~mm} \text { may be necessary } \\
\text { in obese patients }\end{array}$ \\
\hline Slice increment & $\begin{array}{l}\leq \text { slice thickness; overlapping reconstructions not } \\
\text { mandatory }\end{array}$ & $\begin{array}{l}\leq \text { slice thickness, maximum } 0.7 \mathrm{~mm} \text {; overlapping } \\
\text { reconstructions not mandatory }\end{array}$ \\
\hline
\end{tabular}

* should be set with other technical parameters to achieve CTDIvol specifications. ACR-STR, American College of Radiology-Society of Thoracic Radiology; ESTI, European Society of Thoracic Imaging; FBP, filtered-back projection; FOV, field of view; IR, iterative reconstruction; MDCT, multi-detector computed tomography; MIP, maximum intensity projections; MPR, multi-planar reconstruction.

filter. Reduction of the output would lead to decreased image quality and therefore these techniques are often applied in a protocol with iterative reconstruction.

\section{Decreasing tube current and iterative reconstruction}

In the dose reduction studies, the reference LDCT protocols, CT systems and iterative reconstruction algorithms differ considerably (39-44). In the study of Lim et al. a high-pitch scan mode with iterative reconstruction at $120 \mathrm{kVp}$ with fixed tube current of $30 \mathrm{mAs}$ resulted in $34 \%$ dose reduction (2.1 vs. $3.1 \mathrm{mGy})$ with similar image quality and reduced cardiac motion artifacts compared to reference low-dose chest CT at $120 \mathrm{kVp}$ with FBP and low pitch (44). Nevertheless, a fixed tube current of $30 \mathrm{mAs}$ was used in 30 healthy volunteers and reconstructions were made at 3.0/3.0 slice thickness and increment, making the study set-up less suitable to determine the impact on lung nodule evaluation.

In a study of Ye et al. the authors specifically investigated the sensitivity for nodule detection of an ULDCT protocol compared to LDCT protocol in 188 individuals (39). The ULDCT was performed at $120 \mathrm{kVp}$ with $10 \mathrm{~mA}$ while the LDCT was executed at $120 \mathrm{kVp}$ with $50 \mathrm{~mA}$. In both protocols IR $50 \%$ [adaptive statistical iterative 


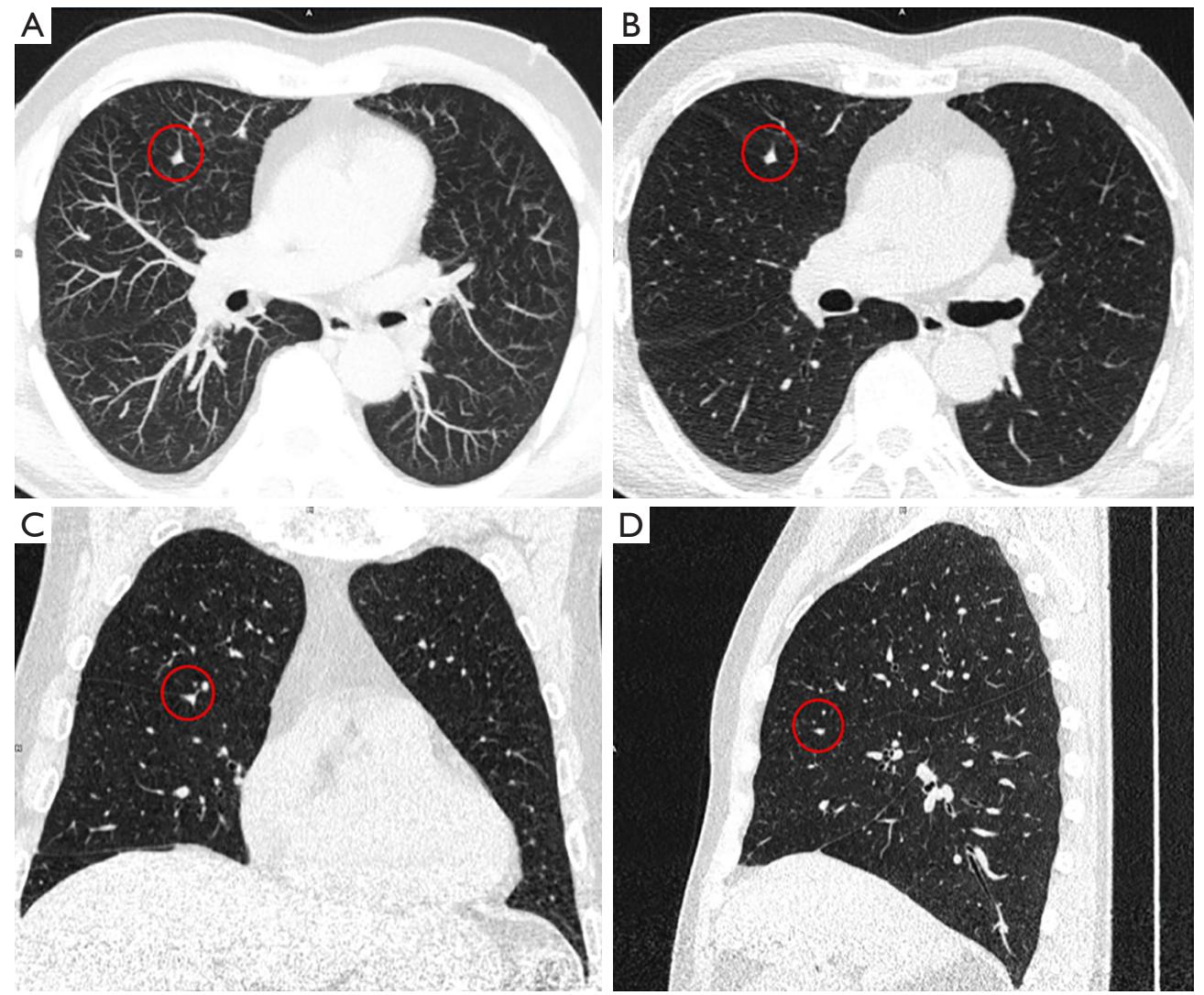

Figure 2 Example of the use of (A) maximum intensity projection (MIP, thickness $10 \mathrm{~mm}$ ) and (B,C,D) multiplanar reconstructions (MPR, thickness) for lung nodule detection and nodule characterization in a patient presenting with a perifissural nodule (highlighted in red circle) of $80 \mathrm{~mm}^{3}$ in the right middle lobe.

reconstruction $\mathrm{V}(\mathrm{ASiR}-\mathrm{V})$ ] was applied, and images were reconstructed at slice thickness and increment of $2.5 \mathrm{~mm}$. The authors found that nodule type (solid, sub-solid) and nodule size (diameter) had influence on the sensitivity. For example, the sensitivity for nodule detection was overall $90.4 \%$ while this could be increased to $98.2 \%$ if only nodules with diameter of $\geq 6 \mathrm{~mm}$ were considered. Although the dose could be reduced from 0.93 to $0.096 \mathrm{mSv}$ based on ULDCT, images were reconstructed at relatively thick slice thickness. Reducing the slice thickness to the preferred $1.0 \mathrm{~mm}$ may drastically impede image quality and sensitivity for nodule detection. Nevertheless, in a study of Zhang et al. ULDCT protocols with IR [iterative model reconstruction (IMR)] and with thin slices were used $(1.0 \mathrm{~mm})$. This study showed that the use of ULDCT with IMR resulted in similar nodule detectability as the reference LDCT with FBP and with hybrid IR, while diagnostic image quality for lung and mediastinum was preserved. However, the ULDCT protocol in this study was with
$0.67 \mathrm{mSv}$ considerably higher than the former study, while obese patients were excluded (42).

\section{Tin-filter and iterative reconstruction}

Several CT systems from one vendor are equipped with a tin filter than can be applied to block the low-energy part of the X-ray spectrum. This part of the spectrum would otherwise have been absorbed by the patient's body and would not have contributed to the image quality but would have increased the total radiation dose.

In a phantom study from Martini et al. the sensitivity of a low-dose tin filter protocol combined with IR [advanced modeled iterative reconstruction (ADMIRE) level 5] for solid and sub-solid nodule detection was determined (45). Sensitivity was $100 \%$ and $93.8 \%$ for solid and sub-solid nodules ranging in size from $2-10 \mathrm{~mm}$ at dose of $0.10 \mathrm{mGy}$. With a slightly higher dose $(0.30 \mathrm{mGy})$, by increasing the tube current from 30 to $80 \mathrm{mAs}$, also a sensitivity of 
$100 \%$ for sub-solid nodules could be reached. In addition, in a phantom study of Eberhard et al. the impact of low dose tin filter protocol on volume measurements of solid nodules was determined. This study showed similar results for standard dose CT and the tin filter protocol for nodule volume ranging from $34-524 \mathrm{~mm}^{3}$ (diameter 4-10) (46). However, absolute percentage error increased for images reconstructed with higher levels of IR (ADMIRE 3 and 5) compared to FBP. This may affect calculation of volume doubling times and consequently impact the clinical management. Nonetheless, volume measurement of solid nodules may still be reliably performed by using a Sn100kVp protocol with FBP at CTDI ${ }_{\text {vol }}$ of $0.10 \mathrm{mGy}$.

In a patient study of Messerli et al. an almost similar CT protocol was applied as in the phantom study of Martini et al., namely Sn $100 \mathrm{kVp}$ with IR (ADMIRE level 3) at $70 \mathrm{mAs}$ (47). The ultra low-dose Sn protocol was applied in 202 patients and sensitivity and image quality were compared to the full dose protocol with $110 \mathrm{kVp}$ and IR (ADMIRE level 3). This patient study showed slightly lower sensitivity of $97.3 \%$ and $92.6 \%$ for all nodules types and sub-solid nodules of $>5 \mathrm{~mm}$, respectively, compared to the phantom study. However, the majority of false negative nodules on ultra low-dose scans had diameter of $\leq 2.0 \mathrm{~mm}$ on the full dose scan, which is well below the lowest size threshold in nodule management protocols. Although the image quality was significantly lower in the ultralow dose protocol this seemed not to affect the detection of relevant lung nodules. Therefore, the radiation dose could be reduced from 3.9 to $0.3 \mathrm{mGy}$ by applying tin-filter protocol with IR. Nonetheless, the impact of the ultralow dose protocol on diameter or volume measurement error was not evaluated in this patient study.

To conclude, the radiation dose may be further decreased beyond the currently recommended guidelines, by using iterative reconstruction and a low tube current or tin-filter. However, the majority of studies investigated only the impact on nodule detection based on certain diameter size with cut-off values varying between studies. Also, only a few studies specified sensitivity by nodule type and many studies excluded obese patients. Therefore, one should be cautious in reducing the radiation to ultralow dose settings.

\section{Future CT protocols \& new developments}

The current guidelines are mainly based on evidence and experience form clinical trials and studies in the past. Continuous efforts are made by the European
Imaging Biomarker Alliance (EIBALL) and Quantitative Imaging Biomarker Alliance (QIBA) to streamline the use and validation of imaging biomarkers like lung nodule quantification in lung cancer CT screening. The goal of these subcommittees of the ESR and RSNA is international standardization and harmonization of data acquisition (CT protocol) and analysis (quantification of lung nodules) $(30,48)$. In particular, the QIBA provides some standards for image quality assurance, rather than specified scan parameter values for LDCT. For instance, minimum requirements for image quality defined by the resolution, edge enhancement, HU deviation, voxel noise and spatial wrapping are defined in the 'QIBA Profile: 'Small Lung Nodule Volume Assessment and Monitoring in Low Dose CT Screening'. New (ultra) low dose CT protocols then may not only be tested against the full dose protocol for nodules' sensitivity but could also be benchmarked against these general image quality parameters.

Besides the image quality assurance with the QIBA profile, the EIBALL keeps track of new developments like computer-aided detection (CAD), radiomics and deep learning in the field of lung cancer (48). The CT protocol can also have impact on the performance of these new applications. In general, use of CAD as a second reader has been found to improve the sensitivity for lung nodule detection (49). This has also shown to be beneficial in ULDCT. In a study of Takahashi et al. 55 patients underwent ULDCT with a tin-filter protocol. Four thoracic radiologists, with 5 to 21 years of experience, analyzed the low dose scans without and once with the help of CAD as a second-reader for the detection of solid, sub-solid and ground glass nodules of $\geq 5 \mathrm{~mm}$ (50). The sensitivity could be increased with $19 \%$ and $20 \%$ for two readers with the help of $\mathrm{CAD}$, whereas for the two others readers the sensitivity was unchanged. In a study by Nomura $e t$ al. the performance of CAD software in ULDCT could be improved by using IR algorithms (51). Training of the CAD software on ULDCT data sets with IR may even further improve the performance of $\mathrm{CAD}$, since the current $\mathrm{CAD}$ software was only trained on scans from low-dose protocols.

Another developing field in which the CT protocol plays an important role is radiomics. In the setting of lung cancer screening, numerous other features besides size and shape can be extracted and analyzed from the acquired CT images, that may allow differentiation between benign and malignant nodules (52). So far, the impact of the CT protocol on radiomic features for nodule characterization has been investigated in a couple of studies. In a phantom 
and patients study by Lo et al. the effects of dose levels, IR levels and reconstruction kernels on density and texture based features of nodules were analyzed (53). Density based feature like histogram mean was the most robust feature, while other features were impacted differently by different dose level and reconstruction. The susceptibility of features for protocol changes should be taken into account when density and texture features are used to characterize nodules or nodule change. If disregarded, one might actually measure protocol settings instead of nodule characteristics. Consequently, the authors argue that $\mathrm{CT}$ protocol settings should be carefully controlled and only robust features used in quantification and characterization of nodules. Kim et al. investigated the impact of other protocol settings like slice thickness, tube current, and reconstruction kernel using a phantom with spherical nodules (54). This study showed that all scan parameters significantly affected almost all of the twenty features that were analyzed. Contrary to the former study, the authors of this study recommend standardization and/or normalization of the features that are extracted from scans acquired with different CT protocols. In a patient study by Choe et al. an attempt was made to normalize scans by deep learning image conversion of chest CT scans to improve reproducibility of radiomic features between soft and hard kernels (55). They showed that the reproducibility of radiomic features could be increased from $15.2 \%$ to $57.4 \%$ if image conversion was used in data set containing scans with different reconstruction kernels. However, reproducibility of features based on the same kernel scans for two readers was significantly higher with $84.3 \%$. The authors conclude that soft and sharp kernels cannot be used interchangeably and the same reconstruction kernel is required to warrant high reproducibility of radiomic features although deep learning image conversion increased the reproducibility considerably.

New deep learning-based algorithms may not only facilitate standardization based on kernel variation, but also allow to improve the image quality of ULDCT. Research in a phantom has found no significant differences in nodule volume were found based on volume measurements on deep learning post-processed images from ULDCT and the physical nodule volume (56). Others studies have shown that deep learning denoising techniques can increase image quality compared to FBP and iterative reconstructed ULDCT images and can preserve structural details in the CT image $(57,58)$. The high image noise present in ULDCT can thus be reduced by these new algorithms, beyond the vendor specific iterative reconstruction techniques, while maintaining image resolution and contrast. This will enable to apply ULDCT in a wider range of screening participants and assists in standardization of CT image quality across CT systems of different vendors.

Currently, the International Association for the Study of Lung Cancer (IASLC) is developing the Early Lung Imaging Confederation (ELIC), with the goal to serve as an global open-source cloud with LDCT images (59). The ELIC cloud will include high-quality thoracic CT images, that have passed the standardized image quality processes. These high-quality images may then be used in global quantitative lung imaging studies to improve the reliability of clinical decision support in CT lung cancer screening and beyond. The new developments described in this review are very promising to accelerate and optimize the accuracy and precision of lung nodule quantification. After further research, these aspects will have to be included in the standardized protocols and recommendations for lung cancer screening. Recommendations from European, US, and global organizations that include these developments are to be awaited.

\section{Conclusions}

In the past two decades, CT lung cancer screening protocols have evolved from prefixed settings for tube voltage and tube current towards automatic tube current modulation and tube voltage selection. Current clinical guidelines provide detailed information on reconstruction settings and encourage the use of thin slices, MPR and MIP for nodule detection and quantification. Based on the guidelines, the CT protocol should result in radiation dose of $<3 \mathrm{mGy}$ for standard size patient. Although cautiously, radiation dose may be further decreased in ultra-low dose CT protocols with the use of IR and tin-filter to $0.3 \mathrm{mGy}$. Performance of new ultra-low dose protocols should be evaluated thoroughly. The sensitivity in relation to nodule size, density and composition should be determined. In addition, impact on volume measurement and reproducibility, as well as performance of $\mathrm{CAD}$ and radiomic features should be evaluated. For the latter, re-training and re-evaluation may be required for optimal use of these techniques in ultra-low dose protocols for lung cancer CT screening. Deep learning denoising techniques are promising to increase the image quality and to apply ultra-low dose CT protocols in a wider range of screening participants, and help to optimize the accuracy and precision of lung nodule quantification and management. With the expected large-scale implementation 
of lung cancer screening, further standardization of (ultra) low-dose CT techniques, including acquisition, postprocessing and reporting, will be required.

\section{Acknowledgments}

Funding: None.

\section{Footnote}

Provenance and Peer Review: This article was commissioned by the Guest Editor (Witold Rzyman) for the series "Implementation of CT-based screening of lung cancer" published in Translational Lung Cancer Research. The article has undergone external peer review.

Conflicts of Interest: All authors have completed the ICMJE uniform disclosure form (available at http://dx.doi. org/10.21037/tlcr-20-808). The series "Implementation of CT-based screening of lung cancer" was commissioned by the editorial office without any funding or sponsorship. Dr. MV reports other from IDNA B.V., outside the submitted work. Dr. RV reports grants from Siemens Healthineers, outside the submitted work. The other authors have no conflicts of interest to declare.

Ethical Statement: The authors are accountable for all aspects of the work in ensuring that questions related to the accuracy or integrity of any part of the work are appropriately investigated and resolved.

Open Access Statement: This is an Open Access article distributed in accordance with the Creative Commons Attribution-NonCommercial-NoDerivs 4.0 International License (CC BY-NC-ND 4.0), which permits the noncommercial replication and distribution of the article with the strict proviso that no changes or edits are made and the original work is properly cited (including links to both the formal publication through the relevant DOI and the license). See: https://creativecommons.org/licenses/by-nc-nd/4.0/.

\section{References}

1. Rzyman W, Szurowska E, Adamek M. Implementation of lung cancer screening at the national level: Polish example. Transl Lung Cancer Res 2019;8:S95-105.

2. Kim Y. Implementation of organized lung cancer screening program in Korea. Ann Oncol 2019;30:ii14.
3. Copeland A, Criswell A, Ciupek A, et al. Effectiveness of Lung Cancer Screening Implementation in the Community Setting in the United States. J Oncol Pract 2019;15:e607-15.

4. Du Y, Li Q, Sidorenkov G, et al. Computed Tomography Screening for Early Lung Cancer, COPD and Cardiovascular Disease in Shanghai: Rationale and Design of a Population-based Comparative Study. Acad Radiol 2021;28:36-45.

5. Du Y, Zhao Y, Sidorenkov G, et al. Methods of computed tomography screening and management of lung cancer in Tianjin: design of a population-based cohort study. Cancer Biol Med 2019;16:181-8.

6. National Targeted Lung Cancer Screening Program Enquiry. Cancer Australia 2019. Available online: https:// canceraustralia.gov.au/about-us/lung-cancer-screeningenquiry

7. Rotterdam EUMC. 4-IN THE LUNG RUN: towards INdividually tailored INvitations, screening INtervals, and INtegrated co-morbidity reducing strategies in lung cancer screening. Cordis EU Research. 2020. Available online: https://cordis.europa.eu/project/id/848294

8. National Lung Screening Trial Research Team, Aberle DR, Adams AM, et al. Reduced Lung-Cancer Mortality with Low-Dose Computed Tomographic Screening. N Engl J Med 2011;365:395-409.

9. de Koning HJ, Van Der Aalst CM, De Jong PA, et al. Reduced lung-cancer mortality with volume CT screening in a randomized trial. N Engl J Med 2020;382:503-13.

10. Heuvelmans MA, Oudkerk M. Appropriate screening intervals in low-dose CT lung cancer screening. Transl Lung Cancer Res 2018;7:281-7.

11. Wood DE, Kazerooni EA, Baum SL, et al. Lung Cancer Screening, Version 3.2018, NCCN Clinical Practice Guidelines in Oncology. J Natl Compr Canc Netw 2018;16:412-41.

12. Perisinakis K, Seimenis I, Tzedakis A, et al. Radiation burden and associated cancer risk for a typical population to be screened for lung cancer with low-dose CT: A phantom study. Eur Radiol 2018;28:4370-8.

13. Demb J, Chu P, Yu S, et al. Analysis of Computed Tomography Radiation Doses Used for Lung Cancer Screening Scans. JAMA Intern Med 2019;179:1650-7.

14. Rampinelli C, De Marco P, Origgi D, et al. Exposure to low dose computed tomography for lung cancer screening and risk of cancer: Secondary analysis of trial data and riskbenefit analysis. BMJ 2017;356:j347.

15. Saltybaeva N, Martini K, Frauenfelder T, et al. Organ 
Dose and Attributable Cancer Risk in Lung Cancer Screening with Low-Dose Computed Tomography. PLoS One 2016;11:e0155722.

16. Infante M, Lutman FR, Cavuto S, et al. Lung cancer screening with spiral CT. Lung Cancer 2008;59:355-63.

17. National Lung Screening Trial Research Team, Aberle DR, Berg CD, et al. The National Lung Screening Trial: Overview and Study Design. Radiology 2011;258:243-53.

18. Lopes Pegna A, Picozzi G, Mascalchi M, et al. Design, recruitment and baseline results of the ITALUNG trial for lung cancer screening with low-dose CT. Lung Cancer 2009;64:34-40.

19. Pedersen JH, Ashraf H, Dirksen A, et al. The Danish Randomized Lung Cancer CT Screening Trial-Overall Design and Results of the Prevalence Round. J Thorac Oncol 2009;4:608-14.

20. van Klaveren RJ, Oudkerk M, Prokop M, et al. Management of Lung Nodules Detected by Volume CT Scanning. N Engl J Med 2009;361:2221-9.

21. Xu DM, Gietema H, de Koning H, et al. Nodule management protocol of the NELSON randomised lung cancer screening trial. Lung Cancer 2006;54:177-84.

22. Wang Y, De Bock GH, Van Klaveren RJ, et al. Volumetric measurement of pulmonary nodules at low-dose chest CT: Effect of reconstruction setting on measurement variability. Eur Radiol 2010;20:1180-7.

23. Ru Zhao Y, Xie X, de Koning HJ, et al. NELSON lung cancer screening study. Cancer Imaging 2011;11 Spec No A:S79-84.

24. Pastorino U, Rossi M, Rosato V, et al. Annual or biennial CT screening versus observation in heavy smokers: 5-year results of the MILD trial. Eur J Cancer Prev 2012;21:308-15.

25. Becker N, Motsch E, Gross ML, et al. Randomized study on early detection of lung cancer with MSCT in Germany: study design and results of the first screening round. J Cancer Res Clin Oncol 2012;138:1475-86.

26. Baldwin DR, Duffy SW, Wald NJ, et al. UK Lung Screen (UKLS) nodule management protocol: modelling of a single screen randomised controlled trial of low-dose CT screening for lung cancer. Thorax 2011;66:308-13.

27. Field JK, Duffy SW, Baldwin DR, et al. The UK Lung Cancer Screening Trial: a pilot randomised controlled trial of low-dose computed tomography screening for the early detection of lung cancer. Health Technol Assess 2016;20:1146.

28. Aberle DR, Gamsu G, Henschke CI, et al. A Consensus Statement of the Society of Thoracic Radiology. J Thorac
Imaging 2001;16:65-8.

29. Devaraj A, van Ginneken B, Nair A, et al. Use of Volumetry for Lung Nodule Management: Theory and Practice. Radiology 2017;284:630-44.

30. CT Volumetry Technical Committee. QIBA Profile : Small Lung Nodule Volume Assessment and Monitoring in Low Dose CT Screening. Quant Imaging Biomarkers Alliance Publicly Rev Draft QIBA. 2018.

31. American College of Radiology. ACR - STR Practice Guideline for the Performance and Reporting of Lung Cancer Screening Thoracic Computed Tomography. 2019;1076:1-12. Available online: https://www.acr.org/-/ media/ACR/Files/Practice-Parameters/CT-LungCaScr.pdf

32. Adult Lung Cancer Screening Technical Specifications 2014. Available online: https://www.acraccreditation.org/-/ media/ACRAccreditation/Documents/LCS/Lung-CancerScreening-Technical-Specifications.pdf?la=en $</$ eref $>$

33. Revel MP. Chest CT for Lung Cancer Screening Technical standards [Internet]. European society of thoracic imaging; 2020. Available online: https://www. myesti.org/content-esti/uploads/ESTI-LCS-technicalstandards_2019-06-14.pdf

34. AAPM Lung Cancer Screening Protocols 2019 [Internet]. American Association of Physicists in Medicine; 2019. Available online: https://www.aapm.org/pubs/ CTProtocols/documents/LungCancerScreeningCT.pdf

35. Baldwin DR, Callister ME. The British Thoracic Society guidelines on the investigation and management of pulmonary nodules. Thorax 2015;70:794-8.

36. MacMahon H, Naidich DP, Goo JM, et al. Guidelines for Management of Incidental Pulmonary Nodules Detected on CT Images: From the Fleischner Society 2017. Radiology 2017;284:228-43.

37. Bueno J, Landeras L, Chung JH. Updated fleischner society guidelines for managing incidental pulmonary nodules: Common questions and challenging scenarios. Radiographics 2018;38:1337-50.

38. Bankier AA, MacMahon H, Goo JM, et al.

Recommendations for Measuring Pulmonary Nodules at CT: A Statement from the Fleischner Society. Radiology 2017;285:584-600.

39. Ye K, Zhu Q, Li M, et al. A feasibility study of pulmonary nodule detection by ultralow-dose CT with adaptive statistical iterative reconstruction-V technique. Eur J Radiol 2019;119:108652.

40. Miller AR, Jackson D, Hui C, et al. Lung nodules are reliably detectable on ultra-low-dose CT utilising modelbased iterative reconstruction with radiation equivalent to 
plain radiography. Clin Radiol 2019;74:409.e17-22.

41. Hassani C, Ronco A, Prosper AE, et al. ForwardProjected Model-Based Iterative Reconstruction in Screening Low-Dose Chest CT: Comparison With Adaptive Iterative Dose Reduction 3D. AJR Am J Roentgenol 2018;211:548-56.

42. Zhang M, Qi W, Sun Y, et al. Screening for lung cancer using sub-millisievert chest $\mathrm{c} T$ with iterative reconstruction algorithm: Image quality and nodule detectability. Br J Radiol 2018;91:20170658.

43. Sui X, Meinel FG, Song $W$, et al. Detection and size measurements of pulmonary nodules in ultra-low-dose CT with iterative reconstruction compared to low dose CT. Eur J Radiol 2016;85:564-70.

44. Lim HK, Ha HI, Hwang HJ, et al. High-pitch, 120 $\mathrm{kVp} / 30 \mathrm{mAs}$, low-dose dual-source chest CT with iterative reconstruction: Prospective evaluation of radiation dose reduction and image quality compared with those of standard-pitch low-dose chest CT in healthy adult volunteers. PLoS One 2019;14:e0211097.

45. Martini K, Ottilinger T, Serrallach B, et al. Lung cancer screening with submillisievert chest CT: Potential pitfalls of pulmonary findings in different readers with various experience levels. Eur J Radiol 2019;121:108720.

46. Eberhard M, Stocker D, Milanese G, et al. Volumetric assessment of solid pulmonary nodules on ultralow-dose CT: A phantom study. J Thorac Dis 2019;11:3515-24.

47. Messerli M, Kluckert T, Knitel M, et al. Ultralow dose CT for pulmonary nodule detection with chest $\mathrm{x}$-ray equivalent dose - a prospective intra-individual comparative study. Eur Radiol 2017;27:3290-9.

48. deSouza NM, Achten E, Alberich-Bayarri A, et al. Validated imaging biomarkers as decision-making tools in clinical trials and routine practice: current status and recommendations from the EIBALL* subcommittee of the European Society of Radiology (ESR). Insights Imaging 2019;10:87.

49. Vlahos I, Stefanidis K, Sheard S, et al. Lung cancer screening: nodule identification and characterization. Transl Lung Cancer Res 2018;7:288-303.

50. Takahashi EA, Koo CW, White DB, et al. Prospective pilot evaluation of radiologists and computer-aided pulmonary nodule detection on ultra-low-dose CT with tin filtration. J Thorac Imaging 2018;33:396-401.

51. Nomura Y, Higaki T, Fujita M, et al. Effects of Iterative Reconstruction Algorithms on Computer-assisted Detection (CAD) Software for Lung Nodules in Ultralow-dose CT for Lung Cancer Screening. Acad Radiol 2017;24:124-30.

52. Wilson R, Devaraj A. Radiomics of pulmonary nodules and lung cancer. Transl Lung Cancer Res 2017;6:86-91.

53. Lo P, Young S, Kim HJ, et al. Variability in CT lungnodule quantification: Effects of dose reduction and reconstruction methods on density and texture based features: Effects. Med Phys 2016;43:4854-65.

54. Kim YJ, Lee HJ, Kim KG, et al. The Effect of CT Scan Parameters on the Measurement of CT Radiomic Features: A Lung Nodule Phantom Study. Comput Math Methods Med 2019;2019:8790694.

55. Choe J, Lee SM, Do KH, et al. Deep Learning-based Image Conversion of CT Reconstruction Kernels Improves Radiomics Reproducibility for Pulmonary Nodules or Masses. Radiology 2019;292:365-73.

56. Han D, Heuvelmans MA, Pelgrim G, et al. Effect of iterative reconstruction and artificial neural network denoising techniques on lung nodule volumetry in ultralow dose computed tomography: a phantom study (B1008). In: Insights into Imaging. European Society of Radiology; 2019. p. 22.

57. Ahn CK, Heo C, Kim JH. Combined low-dose simulation and deep learning for CT denoising: application in ultralow-dose chest CT. Proc. SPIE 11050, International Forum on Medical Imaging in Asia 2019, 110500E (27 March 2019). Available online: https://www. spiedigitallibrary.org/conference-proceedings-ofspie/11050/2521539/Combined-low-dose-simulation-anddeep-learning-for-CT-denoising/10.1117/12.2521539.full

58. Gholizadeh-Ansari M, Alirezaie J, Babyn P. Deep Learning for Low-Dose CT Denoising Using Perceptual Loss and Edge Detection Layer. J Digit Imaging 2020;33:504-15.

59. Mulshine JL, Avila RS, Conley E, et al. The International Association for the Study of Lung Cancer Early Lung Imaging Confederation. JCO Clin Cancer Inform 2020;4:89-99.
Cite this article as: Vonder M, Dorrius MD, Vliegenthart R. Latest CT technologies in lung cancer screening: protocols and radiation dose reduction. Transl Lung Cancer Res 2021;10(2):11541164. doi: $10.21037 /$ tlcr-20-808 